\title{
Poly(ADP-ribosyl)ation of p53 Contributes to TPEN-Induced Neuronal Apoptosis
}

\author{
Hyun-Lim Kim', Hana Ra', Ki-Ryeong Kim, Jeong-Min Lee, Hana Im, and Yang-Hee Kim*
}

\begin{abstract}
Depletion of intracellular zinc by $N, N, N, N^{\prime}$-tetrakis(2-pyridylmethyl) ethylenediamine (TPEN) induces p53-mediated protein synthesis-dependent apoptosis of mouse cortical neurons. Here, we examined the requirement for poly(ADPribose) polymerase (PARP)-1 as an upstream regulator of p53 in zinc depletion-induced neuronal apoptosis. First, we found that chemical inhibition or genetic deletion of PARP-1 markedly attenuated TPEN-induced apoptosis of cultured mouse cortical neurons. Poly(ADP-ribosyl)ation of p53 occurred starting $1 \mathrm{~h}$ after TPEN treatment. Suggesting the critical role of PARP-1, the TPEN-induced increase of stability and activity of p53 as well as poly(ADP-ribosyl)ation of p53 was almost completely blocked by PARP inhibition. Consistent with this, the induction of downstream proapoptotic proteins PUMA and NOXA was noticeably reduced by chemical inhibitors or genetic deletion of PARP-1. TPEN-induced cytochrome $C$ release into the cytosol and caspase-3 activation were also blocked by inhibition of PARP-1. Taken together, these findings indicate that PARP1 is essential for TPEN-induced neuronal apoptosis.
\end{abstract}

\section{INTRODUCTION}

Zinc ions are required for the proper action of many enzymes, structural proteins, and zinc finger transcription factors (Coleman, 1992). Zinc acts as a neurotransmitter in the central nervous system, where it accumulates in synaptic vesicles at high (i.e., $\mathrm{mM}$ ) concentrations, is released by presynaptic stimulation, and regulates synaptic transmission (Frederickson and Bush, 2001). Under normal conditions, intracellular zinc concentration is tightly regulated at very low (i.e., pM) levels (Bozym et al., 2006). Not only does the accumulation of excess zinc after ischemia, epileptic seizures, and traumatic brain injury lead to neuronal death (Choi and Koh, 1998), but the severe depletion of zinc also induces neuronal apoptosis (Ahn et al., 1998; Lee et al., 2008a; Ra et al., 2009). In addition to neurons, many

Department of Molecular Biology, Sejong University, Seoul 143-747, Korea, ${ }^{1}$ These authors contributed equally to this work.

*Correspondence: yhkim@sejong.ac.kr

Received 2 June, 2014; revised 17 January, 2015; accepted 19 January, 2015; published Onine 20 March, 2015

Keywords: caspase-3, NOXA, p53, poly(ADP-ribose) polymerase, PUMA other cell types also undergo apoptosis after zinc depletion (Makhov et al., 2008; McCabe et al., 1993; Wilson et al., 2006).

Previously, we reported that zinc chelation by $\mathrm{N}, \mathrm{N}, \mathrm{N}^{\prime}, \mathrm{N}^{\prime}-$ tetrakis(2-pyridylmethyl)ethylenediamine (TPEN) induces p53dependent apoptosis in cultured mouse cortical neurons (Ahn et al., 1998; Lee et al., 2008a; Ra et al., 2009). Furthermore, chemical or genetic blockade of p53 markedly attenuates this TPEN-induced neuronal apoptosis by blocking induction of proapoptotic proteins, including p53 up-regulated modulator of apoptosis (PUMA), NOXA, and caspase-11 (Ra et al., 2009). These results suggest that the regulation of certain inducible apoptogenic proteins by p53 plays a key role in neuronal apoptosis (Ahn et al., 1998; Lee et al., 2008a; Ra et al., 2009).

p53 is an unstable protein with a short half-life (Jenkins et al., 1985). Normally, p53 is expressed at low levels in a latent form, which quickly accumulates in response to stress signals such as genotoxins, ultraviolet radiation (UV), or oxidative stress (Blagosklonny, 1997; Gorospe et al., 1998; Zhao et al., 2008). The stabilization of p53 is one of the mechanisms by which its activity is regulated. p53 expression is increased by the induction of transcription, post-transcriptional regulation by microRNA, post-translational stabilization by phosphorylation, methylation, or acetylation, and reduction of protein degradation via regulation of the ubiquitin-proteosome system (Appella and Anderson, 2001; Freeman and Espinosa, 2013; Hock and Vousden, 2014; Kroncke, 2003; Lavin and Gueven, 2006). In particular, p53 has many post-translational modification sites (Lavin and Gueven, 2006). However, although the post-translational regulation of p53 by methylation, acetylation, or phosphorylation has been well studied, the function of poly(ADP-ribosyl)ation (PARylation) of p53 is not fully understood.

PARP-1 and PARylation trigger key steps of apoptosis in nonneuronal cells via multiple pro-apoptotic stimuli (Nargi-Aizenman et al., 2002; Simbulan-Rosenthal et al., 1998; 1999; Wang et al., 1998). PARylation of p53 occurs during the early stages of apoptosis and is followed by p53 accumulation (Simbulan-Rosenthal et al., 1998; 1999; Won et al., 2006). An increased expression of p53 is required for apoptosis (Simbulan-Rosenthal et al., 1998), likely involving the transcriptional activation of pro-apoptotic genes and their interaction with other proteins. However, there are few reports on the possible role of poly(ADP-ribose) polymerase-1 (PARP-1) in early stages of apoptosis. Specifically, PARP-1 is a substrate for caspase-3 (Berger, 1985), and the main function of PARP-1 in apoptosis is as a late-stage regulator that maintains levels of intracellular $\mathrm{NAD}^{+} / \mathrm{ATP}$ for programmed cell death via its cleavage and inactivation (Berger, 1985; Berger and Petzold, 1985; Boulares et al., 1999). 
Previously, we found that p53 stability and activity are related to zinc-depleted neuronal apoptosis (Ra et al., 2009) and that PARP-1 is critical regulator of neuronal death (Kim and Koh, 2002; Lee et al., 2008b). Therefore, in the present study, we examined whether PARP-1 acts as an upstream modulator of post-translational modification (i.e., PARylation) of p53, increasing the stability and activity of p53 and inducing the expression of pro-apoptotic proteins in zinc-depleted neuronal apoptosis.

\section{MATERIALS AND METHODS}

\section{Mouse cortical neuron cultures}

Cultures were prepared from mice at embryonic day 14-15 (Lee et al., 2008a; Ra et al., 2009). Dissociated cortical cells were plated onto poly-L-lysine/laminin-coated plates (Nunc, USA) in plating medium containing Dulbecco's Modified Eagle's Medium (DMEM, GibcoBRL, USA) with $20 \mathrm{mM}$ glucose, $38 \mathrm{mM}$ sodium bicarbonate, $2 \mathrm{mM}$ glutamine, $5 \%$ fetal bovine serum, and $5 \%$ horse serum (Cambrex Corp., East Rutherford, USA). Cells from ten hemispheres were plated per 24-well plate. Cytosine arabinoside $(10 \mu \mathrm{M})$ was added at day in vitro 3 (DIV3) to inhibit astroglial proliferation. For $P A R P-1^{+/+}$and $P A R P-1^{-1}$ cortical neuron cultures, we isolated cells from $P A R P-1^{+1+}$ and PARP-1\% mice (The Jackson Lab, USA).

\section{Exposure to drugs}

On DIV7, cultured neurons were exposed to TPEN (Sigma, USA) for 12-24 h in serum-free Eagle's Minimal Essential Medium (EMEM, GibcoBRL). Before treatment, serum-containing medium was removed by multiple rinses and replaced with serum-free EMEM. The PARP inhibitor nicotinamide (NAM) or 3-aminobenzamide $(A B)$ was added to serum-free EMEM $1 \mathrm{~h}$ before and continuously throughout TPEN treatment.

\section{Estimation of cell death}

First, cell death was estimated by direct counting after propidium iodide $(\mathrm{PI})$ exclusion or by measuring the level of lactate dehydrogenase $(\mathrm{LDH})$ released into the medium from irreversibly damaged cells 12-24 $\mathrm{h}$ after TPEN exposure unless otherwise specified (Ra et al., 2009). For PI staining, $2.5 \mu \mathrm{g} / \mathrm{ml}$ PI dye (Sigma, USA) was added directly to the bathing media $24 \mathrm{~h}$ after TPEN treatment, and cultures were washed with fresh EMEM to remove excessive PI dye 5 min later. Because only cells with plasma membrane damage uptake $\mathrm{PI}$ dye, the number of Pl-stained neurons was considered a measure of drug-induced neuronal damage. For LDH assay, samples of bathing media $(50 \mu \mathrm{l})$ obtained from neuronal cultures $24 \mathrm{~h}$ after TPEN treatment were added to $150 \mu \mathrm{LDH}$ release assay buffer [3.8 $\mathrm{mM}$ sodium pyruvate, $0.3 \mathrm{mg} / \mathrm{ml}$ reduced $\mathrm{NADH}$ in $0.1 \mathrm{M} \mathrm{KPO} 4$ buffer ( $\mathrm{pH}$ 7.5)]. The absorbance of the reaction mixture at $340 \mathrm{~nm}$, an index of NADH concentration, was recorded automatically at 2-s intervals for 5 min using a spectrophotometer (Molecular Devices, USA). LDH concentration was automatically calculated from the slope of the absorbance curve. Each PI-positive cell count or LDH value was scaled to the maximal value $(=100)$ after $24-\mathrm{h}$ exposure to $100 \mu \mathrm{M} \mathrm{N}$-methyl-D-aspartic acid (NMDA) in control cultures. To evaluate apoptotic cell death, cultured neurons were stained with $2 \mu \mathrm{g} / \mathrm{ml}$ Hoechst 33342 (Invitrogen, USA) prior to fluorescence microscopy (EVOS fl, AMG, USA), and cells exhibiting condensed chromatin nuclei were considered apoptotic. All experiments were repeated at least three times using cultures from different platings.
Reverse transcription polymerase chain reaction (RT-PCR) Total RNA was isolated using the High Pure RNA Isolation Kit (Roche, Switzerland) and reverse transcribed to cDNA using the oligo(dT)14 primer (Promega, USA). PCR was performed with primer sets specific for Puma, Noxa, Bax, or Dglyceraldehyde-3-phosphate dehydrogenase (Gapdh). PCR was carried out for 30 cycles $\left(94^{\circ} \mathrm{C}\right.$ for $1 \mathrm{~min}, 60^{\circ} \mathrm{C}$ for $1 \mathrm{~min}$, and $72^{\circ} \mathrm{C}$ for $1 \mathrm{~min}$ ). Primer sequences were designed using published cDNA sequences: Puma (5'-CCATTTCTGGGGCTCCAGGA-3', 5'-TCCTCAGCCCTCCCTGTCAC-3'), Noxa (5'-GAACGCGCCAGTGAACCCAA-3', 5'-CTTTGT CTCCAATCCTCCGG-3'), Bax (5'-TGAGCGAGTGTCTCCGGCGA-3', 5'CACGCGGCCCCAGTTGAAGT-3'), and Gapdh (5'-CTACATGGTCTACATGTTCCAGTATG-3', 5'-AGTTGTC ATGGATGACCTTGG-3').

\section{Western blots}

Cell lysates were prepared in lysis buffer (50 mM HEPES, 150 $\mathrm{mM} \mathrm{NaCl}, 1.5 \mathrm{mM} \mathrm{MgCl}_{2}, 5 \mathrm{mM}$ EDTA, 2 mM EGTA, 1\% Triton $\mathrm{X}-100,0.5 \%$ SDS, $\mathrm{pH} 7.4)$. Thirty micrograms of total protein was separated by SDS-PAGE (10\%) under reducing conditions and immunoblotted with antibodies against P-p53 (Ser15), PUMA, Bax, procaspase-3, cleaved caspase-3 (Cell Signaling Technology Inc., USA), poly (ADP-ribose) polymer (PAR), p53, or NOXA (Millipore, USA). Actin (Sigma) was used as a loading control. For immunoprecipitation, cell lysates were prepared using RIPA lysis buffer (50 mM Tris $\mathrm{HCl}, 150 \mathrm{mM} \mathrm{NaCl}, 1 \%$ NP-40, $0.5 \%$ sodium deoxycholate, $0.1 \%$ SDS) and immuneprecipitated with p53 antibody (\#OP33, Merck, Germany). Immunoprecipitated proteins were analyzed by SDS-PAGE $(10 \%)$ and immunoblotted with poly(ADP-ribose) (PAR) antibody (Merck, Germany).

\section{Immunocytochemistry and confocal microscopy}

Neuronal cultures were fixed in $4 \%$ paraformaldehyde at $4^{\circ} \mathrm{C}$ for $30 \mathrm{~min}$ and permeabilized with $0.2 \%$ Triton X-100. After blocking with normal serum in phosphate-buffered saline, cultures were incubated with cytochrome C antibody (\#556432, BD Bioscience, USA) at $4^{\circ} \mathrm{C}$ overnight. Cultures were washed and incubated with a FITC-conjugated secondary antibody (\#715-095-150, Jackson ImmunoResearch Lab Inc, USA) for 2 h. Microscopic images were observed using a laser scanning microscope (TCS SP5, Leica, Germany).

\section{Caspase-3 enzymatic activity assay}

To detect enzymatic activity of caspase-3, the specific substrate for caspase-3, cleavage of ac-DEVD-amc (Millipore, USA), was measured using a fluorometer (Molecular Devices, USA). Protein lysates $(750 \mu \mathrm{g}$ total proteins) were incubated with $100 \mu \mathrm{M}$ fluorogenic tetrapeptide substrate (ac-DEVD-amc). Each fluorescence value is presented as the fold difference from the mean value of sham controls.

\section{Statistical analysis}

All statistical comparisons were performed using analysis of variance (ANOVA) followed by Bonferroni correction for multiple comparisons. A $P$-value of less than 0.05 was considered statistically significant.

\section{RESULTS}

PARP-1 plays a critical role in TPEN-induced neuronal apoptosis.

PARP-mediated post-translational modification (i.e., PARylation) 
A

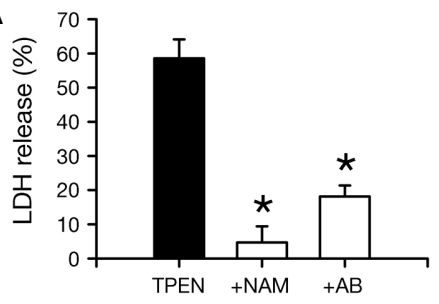

B
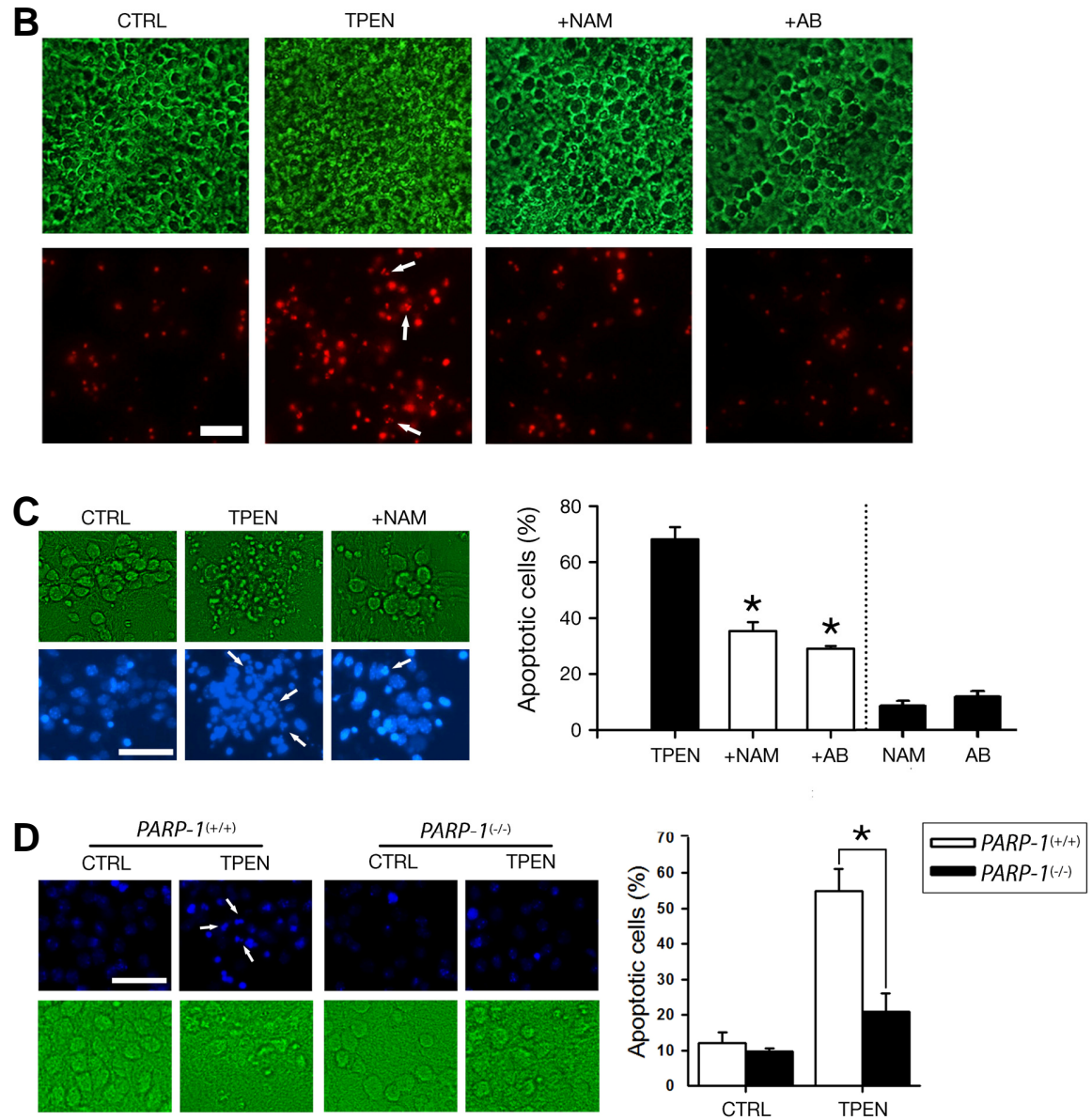

Fig. 1. Requirement for PARP-1 in TPENinduced neuronal apoptosis. (A) LDH release or PI-positive cells (mean \pm SEM, $\mathrm{n}=4$ cultures) in mouse cortical neuron cultures after 24-h exposure to TPEN (2 $\mu \mathrm{M})$ with or without PARP inhibitors nicotinamide (NAM; $10 \mathrm{mM}$ ) or 3-aminobenzamide (AB; $10 \mathrm{mM}$ ). ${ }^{*} p<0.05$ vs. TPEN alone, ANOVA. (B) Phase-contrast (upper) or PI-stained (lower) photomicrographs of identical fields of cultured cortical neurons exposed to sham wash (CTRL) or TPEN with or without NAM or AB for $24 \mathrm{~h}$. Arrows indicate typical apoptotic nuclei. Scale bar $=100 \mu \mathrm{m}$. (C) Photomicrographs (left) and quantitative analysis (right; $n=4$ cultures) of Hoechst 33342-positive apoptotic cells in mouse cortical neuron cultures after 24-h exposure to sham wash (CTRL) or TPEN with or without NAM or AB. Arrows indicate typical morphology of apoptotic condensed nuclei. * $p<0.05$ vs. TPEN alone, ANOVA. (D) Photomicrographs (left) and quantitative analysis (right; $n=4$ cultures) of Hoechst 33342-positive apoptotic cells in $P A R P-1^{+/+}$or PARP-1 $1^{*-}$ mouse cortical neuron cultures after 24-h exposure to sham wash (CTRL) or TPEN. Arrows indicate typical morphology of apoptotic condensed nuclei. In PARP neuronal cultures, zinc-depleted neuronal apoptosis was markedly attenuated. of p53 is required for its rapid accumulation and activation during early stages of apoptosis before commitment to cell death (Simbulan-Rosenthal et al., 1998; 1999). As we previously found that p53 acts as an upstream regulator of TPEN-induced neuronal apoptosis, we examined here whether PARP-1 is essential for p53-mediated protein synthesis-dependent neuronal apoptosis.

To quantify neuronal apoptosis, we used three different methods. First, we measured the release of LDH from neurons into the bathing media. The chemical inhibition of PARP-1 significantly attenuated TEPN-induced neuronal death (Fig. 1A, left). However, some apoptotic insults such as staurosporine do not result in LDH leakage from cell because the process of apoptosis is followed by plasma membrane blebbing rather than rupture. Therefore, we next used PI staining to confirm TPEN-induced neuronal death and its reduction by PARP-1 inhibition. Consistent with our LDH results, the number of $\mathrm{PI}$ - positive neurons was markedly increased by TPEN (Fig. 1A, right; Fig. 1B), and some PI-positive nuclei showed typical shrunken and fragmented morphology (Fig. 1B, arrow). However, this effect was significantly reversed by chemical inhibitors of PARP-1 (Fig. 1A, right; Fig. 1B). To further evaluate apoptotic cell death, we observed the morphology of Hoechst 33342stained nuclei. Whereas TPEN markedly increased the presence of bright and shrunken nuclei, which is a marker of apoptosis, NAM and AB reversed this change in morphology (Fig. $1 C)$. Consistent with these findings, the genetic deletion of PARP-1 almost completely blocked TPEN-induced neuronal apoptosis (Fig. 1D).

Taken together, we found that blockade of PARP-1 by chemical inhibitors (NAM or $\mathrm{AB}$ ) or genetic deletion $(P A R P-1)$ markedly attenuated TPEN-induced death of cultured mouse cortical neurons, indicating that PARP-1 is essential for TPEN-induced neuronal apoptosis. 


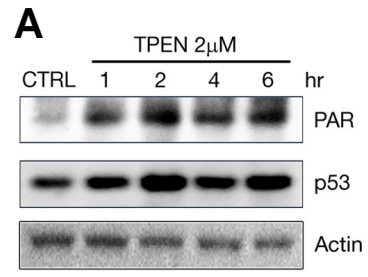

D

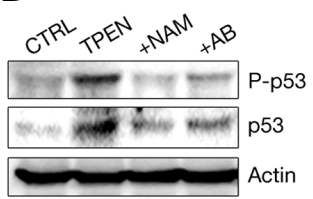

$B$

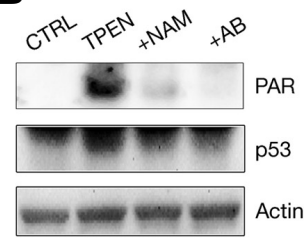

$E$

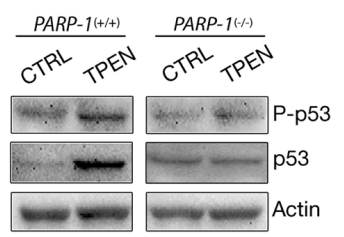

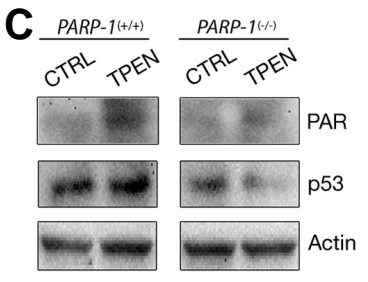

Fig. 2. Post-translational modification of p53 by PARP-1 in TPEN-induced neuronal apoptosis. (A) Immunoprecipitation (IP) and immunoblotting over the time course of p53 PARylation. Protein samples were prepared from nearly pure cortical neuron cultures at the indicated time points after TPEN treatment. Protein extracts were immunoprecipitated with p53 antibody and analyzed by SDS-PAGE and immunoblotting with poly(ADP-ribose) (PAR) antibody. PARylation bands of p53 were detected from $1 \mathrm{hr}$ after TPEN treatment. To show that the same amount of protein extract was used in IP, $30 \mu \mathrm{l}$ of protein $(1 \mu \mathrm{g} / \mu \mathrm{l})$ was prepared before IP incubation and analyzed using actin antibody. (B) IP and immunoblotting for p53 PARylation. Protein samples were prepared after 4-h exposure to TPEN with or without NAM or AB. PARylation of $\mathrm{p} 53$ was almost completely blocked by PARP inhibitors. (C) PARylation of p53 in PARP-1+t or PARP-1 mouse cortical neuron cultures. Protein samples were prepared after 4-h exposure to sham wash (CTRL) or TPEN and then immunoprecipitated with p53 antibody. PARylation and accumulation of p53 by TPEN were not induced in PARP-1 mouse cortical neuron cultures. (D) Western blot analysis of p53 phosphorylation (upper) and accumulation (lower). Protein samples were prepared after 6-h exposure to TPEN with or without NAM or AB. PARP inhibitors markedly attenuated the TPEN-induced increase in p53 activity and stability. (E) Western blot analysis of p53 phosphorylation (upper) and accumulation (lower) in PARP-1 $1^{+/ t}$ or PARP-1/ mouse cortical neuron cultures. Protein samples were prepared after 6 -h exposure to sham wash (CTRL) or TPEN. The increase of p53 activity and stability by TPEN was not detected in PARP-1 deficient neuronal cultures.

$A$

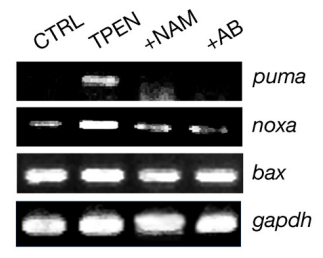

B

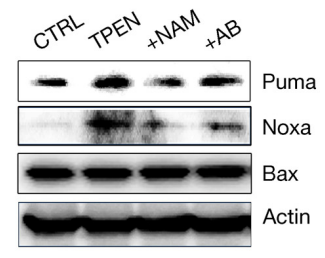

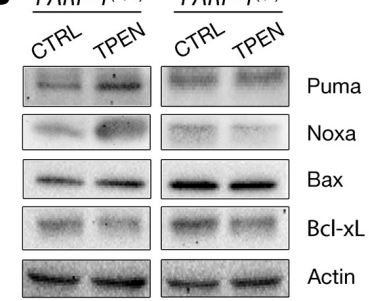

Fig. 3. PARP-1 as an upstream regulator of TPEN-induced pro-apoptotic proteins. (A) RTPCR (left) and western blot analysis (right) of PUMA, NOXA, and Bax expression. RNA or protein samples were prepared from neuron cultures after 6-h exposure to sham wash (CTRL) or TPEN with or without NAM or AB. (B) Western blot analysis of PUMA, NOXA, and Bax expression. Protein samples were prepared from PARP-1+t or PARP-1/ mouse cortical neuronal cultures after 6-h exposure to sham wash (CTRL) or TPEN. Inhibition of PARP-1 by chemical inhibitors or genetic deletion almost completely reduced the induction of proapoptotic proteins by TPEN.

Poly(ADP-ribosyl)ation of p53 by PARP-1 augments the stability and activity of p53

Next, we investigated the PARylation of p53 in TPEN-induced neuronal apoptosis. Substantial PARylation of p53 was detected $1 \mathrm{~h}$ after TPEN treatment (Fig. 2A) and blocked by PARP inhibitors (Fig. 2B). In PARP-1 cortical neuron cultures, PARylation by TPEN was not detected (Fig. 2C). Consistent with this observation, TPEN-induced accumulation and phosphorylation of p53 were markedly attenuated by chemical inhibitors (Fig. 2D) or genetic deletion of PARP-1 (Fig. 2E). These results strongly suggest that PARP-1 regulates the stability and activity of p53 via post-translational modification (i.e., PARylation) in TPEN-induced neuronal apoptosis.

Induction of pro-apoptogenic proteins PUMA and NOXA is mediated by PARP-1 in zinc-depleted neuronal apoptosis We previously showed that $\mathrm{Bcl}-2$ homology domain 3 (BH3)-only proteins, such as PUMA and NOXA, are induced by TPEN in a p53-dependent manner (Ra et al., 2009). Here, we found that increases in $\mathrm{BH} 3-$ only pro-apoptotic proteins were mediated by PARP-1. Induction of PUMA and NOXA expression by TPEN was markedly reduced by chemical inhibitors of PARP (Fig. 3A).
In PARP-1 ${ }^{-1}$ neuronal cultures, the protein expression level of PUMA or NOXA was not increased by TPEN (Fig. 3B), indicating mediation by PARP-1. Bax, expression levels were not affected by TPEN in either PARP-1 $1^{+/ t}$ or PARP-1 $1^{-/}$cortical neuron cultures (Fig. 3B). Consistently, the chemical inhibition or genetic deletion of PARP-1 had no effect on Bax expression levels (Fig. 3B).

Blockade of PARP-1 markedly diminished caspase-3 activation in zinc-depleted neuronal apoptosis

Next, we investigated the effects of PARP-1 on downstream events of TPEN-induced apoptosis, including cytochrome $\mathrm{C}$ release and caspase-3 activation. TPEN-induced cytochrome $\mathrm{C}$ release from mitochondria to the cytosol and nuclear condensation were completely blocked by the PARP inhibitor NAM (Fig. 4A). TPEN-induced caspase-3 activation was also blocked by chemical inhibitors (Figs. 4B-4C) or genetic deletion of PARP-1 (Figs. 4D-4E). Thus, PARP-1 appears to act as an upstream regulator of TPEN-induced neuronal apoptosis.

\section{DISCUSSION}

We demonstrated that PARP-1 is essential for TPEN-induced 
A
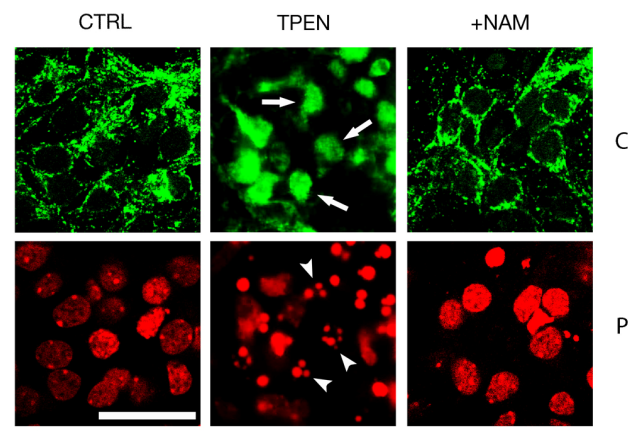

B

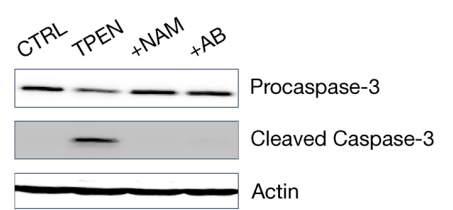

D

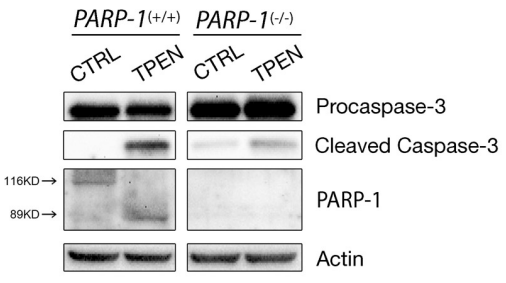

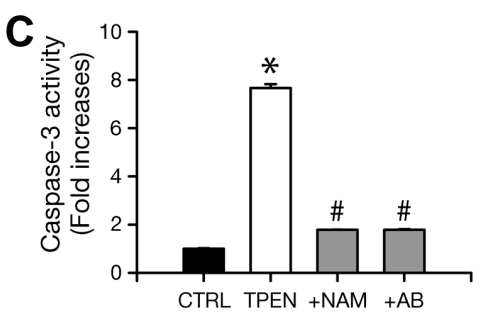

$F$

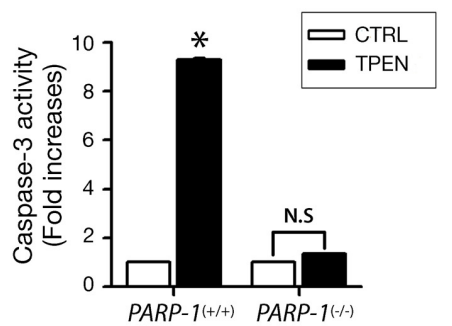

Fig. 4. Essential role of PARP-1 as an upstream regulator of TPEN-induced neuronal apoptosis. (A) Confocal photomicrographs of cortical neuron cultures showing the translocation of cytochrome $\mathrm{C}$ from mitochondria to the cytosol after TPEN treatment. NAM blocked this TPEN-induced release of cytochrome $\mathrm{C}$ into the cytosol. Scale bar $=50 \mu \mathrm{m}$. Arrows and arrowheads denote cytochrome $C$ release and DNA fragmentation, respectively. $(B, C)$ Western blot analysis $(B)$ or enzymatic activity $(C, n=4$ cultures) of caspase-3 in TPEN-induced neuronal apoptosis. Protein samples were prepared after 12-h exposure to sham wash (CTRL) or TPEN with or without NAM or AB. ${ }^{*} p<0.05$ vs. TPEN alone, ANOVA. (D, E) Western blot analysis (D) or enzymatic activity $(E, n=$ 4 cultures) of caspase-3 in PARP-1 $1^{+t}$ or PARP-1 mouse cortical neuron cultures after 18-h exposure to sham wash (CTRL) or TPEN. ${ }^{*} p<0.05$ vs. sham wash control, ANOVA. neuronal apoptosis via the regulation of p53 stability and activity. We previously showed that de novo protein synthesis of PUMA and NOXA is required for TPEN-induced neuronal apoptosis in a p53-dependent manner (Ra et al., 2009). Here, we showed that the chemical inhibition or genetic deletion of PARP-1 almost completely blocked the TPEN-induced increase in p53 stability and activity, induction of PUMA and NOXA, cytochrome $C$ release, caspase- 3 activation, and neuronal death, indicating an essential role for PARP-1 as an upstream regulator in TPEN-induced neuronal apoptosis.

Although we suggest that PARylation of p53 is the main regulatory mechanism of p53 stability and activity when neurons are exposed to zinc depletion stress, we cannot rule out other possible mechanisms. In particular, levels of p53 expression in control neuronal cultures from $P A R P-1^{-1}$ mice were slightly higher than those in control neuronal cultures from $P A R P-1^{+/ t}$ mice (Fig. 2E). In addition, the expression of all $\mathrm{Bcl}-2$ family proteins that were assessed in the present study, including PUMA, NOXA, Bax, and Bcl-xL, was also slightly higher in PARP-1 neuronal cultures (Fig. 3B). Under PARP-1-deficient conditions, the DNA repair process may be damaged or modified, and expression of the tumor suppressor protein p53 and related genes could also be affected by the deletion of PARP-1. Our most important finding, however, is that there was no additional increase in levels of p53 or Bcl-2 family proteins by TPEN in PARP-1\% cortical neuron cultures (Fig. 2E; Fig. 3B). Furthermore, our results suggest that the relative increase in levels of pro-apoptotic Bcl-2 family proteins PUMA and Bax may be compensated by the simultaneous increase of the antiapoptotic Bcl-2 family protein Bcl-xL (Fig. 3B). In other words, the lack of induction of apoptosis under normal conditions in PARP-1 cortical neuron cultures may be due to a balance between pro-apoptotic and anti-apoptotic proteins. Future studies are needed to confirm this possibility.

Among members of the PARP gene family, PARylation is mainly catalyzed by PARP-1, PARP-2, and PARP-3 (Johansson, 1999). PARP-1 has two N-terminal zinc finger motifs and an automodification domain, whereas PARP-2 and PARP-3 have only a C-terminal catalytic domain (Johansson, 1999; Li and Chen, 2014). For PARP-1, displacement of zinc from the zinc finger by other metal ions leads to decreased PARP-1 activity (Mendes et al., 2011). However, the direct displacement of zinc from PARP-1 is likely not an underlying mechanism of TPENinduced neuronal apoptosis, as we showed that PARylation is increased by TPEN and that TPEN-induced neuronal death is due to the chelation of zinc ions rather than other metal ions (Ahn et al., 1998). Some previous studies report that neutral endonuclease activity is regulated by intracellular zinc levels and that the depletion of intracellular free zinc by TPEN increases nuclease activity and DNA degradation (Marini and Musiani, 1998; Villalba et al., 1995; Widlak and Garrard, 2001). Therefore, DNA damage could trigger PARP-1 activation in TPEN-induced neuronal apoptosis. However, further studies are needed to completely understand how PARP-1 is activated in early stages of TPEN-induced apoptosis.

Although we show a requirement for PARP-1 in neuronal 
apoptosis, there is some controversy about the role of PARP-1 in apoptosis. For instance, PJ34, a potent inhibitor of PARP-1, independently kills tumor cells in a caspase-dependent manner (Gangopadhyay et al., 2011). Also, veliparib (ABT-888), another PARP-1 inhibitor, enhances the DNA damage response and increases the death of cancer cells in both p53-dependent and independent manners (Nguyen et al., 2011). These discrepancies among studies could be due to differences among cell populations. Thus, additional studies are required to investigate different actions of PARP-1 in the death of non-dividing neurons and strongly proliferating cancer cells. Because several PARP-1 inhibitors have been developed to potentiate the cytotoxicity of ionizing radiation and anticancer drugs, our results provide important considerations for cancer research and advance our understanding of the mechanisms of neuronal apoptosis.

\section{ACKNOWLEDGMENTS}

This work was supported by National Research Foundation of Korea (NRF) grants from the Korean government (MSIP) (NRF-2009-0081490, NRF-2009-0066905) and a grant from the Korea Health Technology R\&D Project, Ministry of Health \& Welfare, Republic of Korea (A084219).

\section{REFERENCES}

Ahn, Y.H., Kim, Y.H., Hong, S.H., and Koh, J.Y. (1998). Depletion of intracellular zinc induces protein synthesis-dependent neuronal apoptosis in mouse cortical culture. Exp. Neurol. 154, 47-56.

Appella, E., and Anderson, C.W. (2001). Post-translational modifications and activation of p53 by genotoxic stresses. Eur. J. Biochem. 268, 2764-2772.

Berger, N.A. (1985). Poly(ADP-ribose) in the cellular response to DNA damage. Radiat. Res. 101, 4-15.

Berger, N.A., and Petzold, S.J. (1985). Identification of minimal size requirements of DNA for activation of poly(ADP-ribose) polymerase. Biochemistry 24, 4352-4355.

Blagosklonny, M.V. (1997). Loss of function and p53 protein stabilization. Oncogene 15, 1889-1893.

Boulares, A.H., Yakovlev, A.G., Ivanova, V., Stoica, B.A., Wang, G. lyer, S., and Smulson, M. (1999). Role of poly(ADP-ribose) polymerase (PARP) cleavage in apoptosis. Caspase 3-resistant PARP mutant increases rates of apoptosis in transfected cells. J. Biol. Chem. 274, 22932-22940.

Bozym, R.A., Thompson, R.B., Stoddard, A.K., and Fierke, C.A. (2006). Measuring picomolar intracellular exchangeable zinc in $\mathrm{PC}-12$ cells using a ratiometric fluorescence biosensor. ACS Chem. Biol. 1, 103-111.

Choi, D.W., and Koh, J.Y. (1998). Zinc and brain injury. Annu. Rev. Neurosci. 21, 347-375.

Coleman, J.E. (1992). Zinc proteins: enzymes, storage proteins, transcription factors, and replication proteins. Annu. Rev. Biochem. 61, 897-946.

Frederickson, C.J., and Bush, A.I. (2001). Synaptically released zinc: physiological functions and pathological effects. Biometals $14,353-366$.

Freeman, J.A., and Espinosa, J.M. (2013). The impact of posttranscriptional regulation in the p53 network. Brief. Funct. Genomics 12, 46-57.

Gangopadhyay, N.N., Luketich, J.D., Opest, A., Visus, C., Meyer, E.M., Landreneau, R., and Schuchert, M.J. (2011). Inhibition of poly(ADP-ribose) polymerase (PARP) induces apoptosis in lung cancer cell lines. Cancer Invest. 29, 608-616.

Gorospe, M., Wang, X., and Holbrook, N.J. (1998). p53-dependent elevation of p21Waf1 expression by UV light is mediated through mRNA stabilization and involves a vanadate-sensitive regulatory system. Mol. Cell. Biol. 18, 1400-1407.

Hock, A.K., and Vousden, K.H. (2014). The role of ubiquitin modification in the regulation of p53. Biochim. Biophys. Acta 1843, 137-149.

Jenkins, J.R., Rudge, K., Chumakov, P., and Currie, G.A. (1985). The cellular oncogene p53 can be activated by mutagenesis.
Nature 317, 816-818

Johansson, M. (1999). A human poly(ADP-ribose) polymerase gene family (ADPRTL): cDNA cloning of two novel poly(ADPribose) polymerase homologues. Genomics 57, 442-445.

Kim, Y.H., and Koh, J.Y. (2002). The role of NADPH oxidase and neuronal nitric oxide synthase in zinc-induced poly(ADP-ribose) polymerase activation and cell death in cortical culture. Exp. Neurol. 177, 407-418.

Kroncke, K.D. (2003). Nitrosative stress and transcription. Biol. Chem. 384, 1365-1377.

Lavin, M.F., and Gueven, N. (2006). The complexity of p53 stabilization and activation. Cell Death Differ. 13, 941-950.

Lee, J.M., Kim, Y.J., Ra, H., Kang, S.J., Han, S., Koh, J.Y., and Kim, Y.H. (2008a). The involvement of caspase-11 in TPEN-induced apoptosis. FEBS Lett. 582, 1871-1876.

Lee, J.Y., Kim, Y.J., Kim, T.Y., Koh, J.Y., and Kim, Y.H. (2008b). Essential role for zinc-triggered p75NTR activation in preconditioning neuroprotection. J. Neurosci. 28, 10919-10927.

Li, N., and Chen, J. (2014). ADP-ribosylation: activation, recognition, and removal. Mol. Cells 37, 9-16.

Makhov, P., Golovine, K., Uzzo, R.G., Rothman, J., Crispen, P.L. Shaw, T., Scoll, B.J., and Kolenko, V.M. (2008). Zinc chelation induces rapid depletion of the X-linked inhibitor of apoptosis and sensitizes prostate cancer cells to TRAIL-mediated apoptosis. Cell Death Differ. 15, 1745-1751.

Marini, M., and Musiani, D. (1998). Micromolar zinc affects endonucleolytic activity in hydrogen peroxide-mediated apoptosis. Exp. Cell Res. 239, 393-398.

McCabe, M.J., Jr., Jiang, S.A., and Orrenius, S. (1993). Chelation of intracellular zinc triggers apoptosis in mature thymocytes. Lab. Invest. 69, 101-110.

Mendes, F., Groessl, M., Nazarov, A.A., Tsybin, Y.O., Sava, G. Santos, I., Dyson, P.J., and Casini, A. (2011). Metal-based inhibition of poly(ADP-ribose) polymerase--the guardian angel of DNA. J. Med. Chem. 54, 2196-2206.

Nargi-Aizenman, J.L., Simbulan-Rosenthal, C.M., Kelly, T.A., Smulson, M.E., and Griffin, D.E. (2002). Rapid activation of poly(ADP-ribose) polymerase contributes to Sindbis virus and staurosporine-induced apoptotic cell death. Virology 293, 164-171.

Nguyen, D., Zajac-Kaye, M., Rubinstein, L., Voeller, D., Tomaszewski, J.E., Kummar, S., Chen, A.P., Pommier, Y., Doroshow, J.H., and Yang, S.X. (2011). Poly(ADP-ribose) polymerase inhibition enhances p53-dependent and -independent DNA damage responses induced by DNA damaging agent. Cell Cycle 10, 40744082.

Ra, H., Kim, H.L., Lee, H.W., and Kim, Y.H. (2009). Essential role of p53 in TPEN-induced neuronal apoptosis. FEBS Lett. 583, 1516-1520.

Simbulan-Rosenthal, C.M., Rosenthal, D.S., lyer, S., Boulares, A.H., and Smulson, M.E. (1998). Transient poly(ADP-ribosyl)ation of nuclear proteins and role of poly(ADP-ribose) polymerase in the early stages of apoptosis. J. Biol. Chem. 273, 13703-13712.

Simbulan-Rosenthal, C.M., Rosenthal, D.S., Luo, R., and Smulson, M.E. (1999). Poly(ADP-ribosyl)ation of p53 during apoptosis in human osteosarcoma cells. Cancer Res. 59, 2190-2194.

Villalba, M., Ferrari, D., Bozza, A., Del Senno, L., and Di Virgilio, F. (1995). Ionic regulation of endonuclease activity in PC12 cells. Biochem. J. 311 (Pt 3), 1033-1038.

Wang, X., Ohnishi, K., Takahashi, A., and Ohnishi, T. (1998) Poly(ADP-ribosyl)ation is required for p53-dependent signal transduction induced by radiation. Oncogene 17, 2819-2825.

Widlak, P., and Garrard, W.T. (2001). Ionic and cofactor requirements for the activity of the apoptotic endonuclease DFF40/CAD. Mol. Cell. Biochem. 218, 125-130.

Wilson, D., Varigos, G., and Ackland, M.L. (2006). Apoptosis may underlie the pathology of zinc-deficient skin. Immunol. Cell Biol. 84, 28-37.

Won, J., Chung, S.Y., Kim, S.B., Byun, B.H., Yoon, Y.S., and Joe, C.O. (2006). Dose-dependent UV stabilization of p53 in cultured human cells undergoing apoptosis is mediated by poly(ADPribosyl)ation. Mol. Cells 21, 218-223.

Zhao, J., Chen, J., Lu, B., Dong, L., Wang, H., Bi, C., Wu, G., Guo, H., Wu, M., and Guo, Y. (2008). TIP30 induces apoptosis under oxidative stress through stabilization of p53 messenger RNA in human hepatocellular carcinoma. Cancer Res. 68, 4133-4141. 